\title{
AFLP-SSCP: A Useful AFLP-Based Method for Informative SNPs Discovery in Non-Model Organisms
}

\author{
Te-Hua Hsu', Yue Ning², Jin-Chywan Gwo ${ }^{1 *}$ \\ ${ }^{1}$ Department of Aquaculture, National Taiwan Ocean University, Taiwan \\ ${ }^{2}$ Fisheries Research Institute of Fujian, Xiamen, China \\ Email: "gwojc@hotmail.com
}

Received 18 July 2014; revised 6 September 2014; accepted 23 September 2014

Copyright (C) 2014 by authors and Scientific Research Publishing Inc.

This work is licensed under the Creative Commons Attribution International License (CC BY). http://creativecommons.org/licenses/by/4.0/

c) (i) Open Access

\begin{abstract}
Single nucleotide polymorphisms (SNPs) are the most common type of genetic variation among individuals of a species. Recently, in spite of the development of high-throughput genotyping technologies, SNPs have been applied as markers for population genetic and high-density genetic mapping. However, the high costs of SNPs discovery and genotyping assay limit the applications of SNP markers in non-model organisms. In this study, we present a cheap and convenient AFLPbased (Amplified fragment length polymorphism) strategy that is highly efficient for developing informative SNP markers without any prior information. We developed SNP markers in a nonmodel and economic aquaculture species Asian Seabass (Lates calcarifer), and discussed the potential use of the combinations of AFLP and AFLP-SSCP.
\end{abstract}

\section{Keywords}

AFLP, SNPs, Molecular Marker, SSCP

\section{Introduction}

Single nucleotide polymorphisms (SNPs), the most common type of genetic variation among individuals of a species, were considered as powerful markers for genetic mapping and genome-wide association analysis [1]-[3]. Recently, in spite of the development of high-throughput genotyping technologies, more and more studies had employed SNPs in various researches; however, only focused in model species [3]. Non-model organisms, always lack of genome information, were difficult to develop enough SNP markers to use. The high costs of SNPs

${ }^{*}$ Corresponding author. 
discovery and genotyping assay limit the applications of SNP markers [1] [3] [4]. To find cheap and highly efficient method for SNPs discovery and genotyping assay is necessary [3] [4].

Despite the limitations of SNP marker in non-model organisms, the AFLP (amplified fragment length polymorphism) has proven to be a previously useful tool. Due to the advantages of no prior information required, universal and modifiable protocol and a large number of markers per analysis, AFLP has been widely applied in plants, fungi, bacteria and animals [5] [6]. AFLP is ideal for non-model organisms in various short-term researches such as species (strain, subspecies or hybrids) identification, population genetic, shallow phylogenetic reconstructions and genetic mapping [5]-[7].

Although AFLP shows many advantages, it still has some prior shortcomings for the further applications such as the dominant marker, not locus-specific and hardly comparable. In order to save this problem, many studies have tried to converse AFLP markers into simple PCR markers (ex. STS, CAPS or dCAPS markers) [8]-[10]. Nevertheless, this conversion efficiency severely reduced when AFLP markers that typically involve SNPs in the restriction sites or selective primer sites. It will be difficult to design new pairs for locus-specific amplification from the unknown flanking sequence [10] [11].

Nicod and Largiader (2003) [4] developed a SNP isolation strategy that consists of direct sequencing of AFLP bands. Many polymorphic AFLP bands were sequenced without cloning. It provided a rich resource for the SNPs discovering. However, in Nicod and Largiader (2003) [4] study, there is low efficiency that only 10 of the 29 successfully sequenced bands (34\%) contained SNPs.

In this study, we present an AFLP-based SNPs discovery strategy that is highly efficient and economic for developing informative SNP markers without any prior information. Additionally, this method can exclude SNPs that involved in the restriction sites or selective primer sites, and increase the conversion efficiency. We used Single-strand conformation polymorphism (SSCP) to detect SNPs from monomorphic AFLP bands, and named it AFLP-SSCP. This method was examined in Asian Seabass (Lates calcarifer), a non-model and economic aquaculture species, for stock specific SNPs development.

\section{Material and Methods}

\subsection{Sample Collection and DNA Extraction}

The Asian Seabass samples used in this study were collected from different stock in Taiwan (10 specimens), Indonesia (20 specimens) and Thailand (15 specimens), respectively. Ethanol-preserved tissue samples were stored at $-20^{\circ} \mathrm{C}$ until genomic DNA could be isolated from the dorsal fin of each sample by standard phenolchloroform method. DNA concentration was measured with an UV spectrophotometer (NanoDrop ND-1000, Thermo, USA). The quality of extracted DNA was assessed by $1.0 \%$ agarose gel electrophoresis with ethidium bromide.

\subsection{AFLP Reactions}

AFLP reactions were performed as Vos et al. (1995) [12] and Wang et al. (2004) [13] scribed. Initially, about $100 \mathrm{ng}$ of total genomic DNA was digested with $5 \mathrm{U}$ of EcoRI and Tru9I (Promega, USA) in buffer $\mathrm{C}$ at $37^{\circ} \mathrm{C}$ and $65^{\circ} \mathrm{C}$ for $3 \mathrm{~h}$, respectively. Then, the digested DNA fragments were ligated with $2.5 \mathrm{pmol}$ of EcoRI and 25 pmol Msel adapters in a reaction mixture containing 0.25 mg BSA, 5 pmol ATP, 0.04 U T4 DNA ligase (Promega, USA) and $10 \times$ ligation buffer at $37^{\circ} \mathrm{C}$ for $3 \mathrm{~h}$. The pre-selective amplification was conducted on $50 \mathrm{ng}$ of ligation products in 20-ml reactions with the following composition: $1 \times$ Go Taq Flexi buffer, $2.0 \mathrm{mM} \mathrm{MgCl}$, $0.2 \mathrm{mM}$ each dNTPs, 2 pmol each of pre-selective primers (EcoRI-A and MseI-C) and $0.1 \mathrm{U}$ Taq (Promega). Cycling parameters were $94^{\circ} \mathrm{C}$ for $2 \mathrm{~min}$, followed by 30 cycles of $94^{\circ} \mathrm{C}$ for $30 \mathrm{~s}, 56^{\circ} \mathrm{C}$ for $1 \mathrm{~min}$, and $72^{\circ} \mathrm{C}$ for 1 min, followed by $72^{\circ} \mathrm{C}$ for 2 min. The PCR product was diluted $1: 20$ with distilled water and used as templates for the subsequent selective PCR amplification. In the selective amplification, each reaction contained $1 \mathrm{ul} \mathrm{di-}$ luted pre-selective product, $1 \times$ Go Taq Flexi buffer, $1.6 \mathrm{mM} \mathrm{MgCl}_{2}, 0.2 \mathrm{mM}$ each dNTPs, 2 pmol each of selective primers (EcoRI-ANN and MseI-CNN) and $0.1 \mathrm{U}$ Taq (Promega). The PCR conditions were: $94^{\circ} \mathrm{C}$ for 2 min, $65^{\circ} \mathrm{C}$ for $30 \mathrm{~s}, 72^{\circ} \mathrm{C}$ for $1 \mathrm{~min}, 1$ cycle; $94^{\circ} \mathrm{C}$ for $30 \mathrm{~s}, 65^{\circ} \mathrm{C}-56^{\circ} \mathrm{C}$ for $30 \mathrm{~s}\left(-1^{\circ} \mathrm{C} /\right.$ cycle), and $72^{\circ} \mathrm{C}$ for $1 \mathrm{~min}, 10$ cycles; $94^{\circ} \mathrm{C}$ for $30 \mathrm{~s}, 56^{\circ} \mathrm{C}$ for $30 \mathrm{~s}$, and $72^{\circ} \mathrm{C}$ for $1 \mathrm{~min}, 26$ cycles. The selective amplification was performed using seven pairs of primer sets, E-ACG/M-CTC; E-AGT/M-CCG; E-ATC/M-CGT; E-ACT/M-CGC; E-ATT/ M-CGA; E-AAT/M-CCG; and E-ATG/M-CTA. 


\subsection{Electrophoresis and Silver Staining}

The PCR products were mixed with $6 \times$ loading dyes (99\% formamide, 10 mM EDTA, $0.05 \%$. bromophenol and $0.05 \%$ xylene cyanol). The product mixtures were denatured and concentrated at $94^{\circ} \mathrm{C}$ for $10 \mathrm{~min}$, and quickly cooled in an ice bath after denaturation. For standard AFLP, a 5\% denaturing polyacrylamide gel (4.75\% acrylamide, $0.25 \%$ bisacrylamide, $7 \mathrm{M}$ urea and $1 \times \mathrm{TBE}$ ) was prerun at $1800 \mathrm{~V}$ for $30 \mathrm{~min}$. Each well was loaded with $1.0 \mu \mathrm{l}$ of sample. The gel was electrophoresed for $2.5 \mathrm{~h}$ in an ATTO (Type AE6155, Tokyo, Japan) DNA sequencing cell $(38 \times 50 \mathrm{~cm})$ at $1800 \mathrm{~V}$ and $50^{\circ} \mathrm{C}$. For AFLP-SSCP, a $6 \%$ non-denaturing polyacrylamide gel (4.875\%, $0.125 \%$ bisacrylamide, $5 \%$ glycerol and $1 \times$ TBE) was used at as prerun at $300 \mathrm{~V}$ for $30 \mathrm{~min}$. The gel was electrophoresed for $16 \mathrm{~h}$ at $300 \mathrm{~V}$ and room temperature $\left(25^{\circ} \mathrm{C}\right)$. After electrophoresis, the gel was fixed in $1 \%$ ethanoic acid for at least $30 \mathrm{~min}$. The gel was rinsed in distilled water and stained with a mixture of $0.1 \%$ silver nitrate and $0.007 \%$ benzene sulphonic acid for $30 \mathrm{~min}$. The stained gel was rinsed again with distilled water and immersed in a developing solution (2.5\% sodium carbonate, $0.037 \%$ formaldehyde and $0.002 \%$ sodium thiosulphate). The development was subsequently stopped with $1 \%$ ethanoic acid when bands were visible and reached desirable intensity. Band sizes were estimated using a standard AFLP.

\subsection{Band Re-Amplification and Sequencing}

Comparison of AFLP-SSCP profile with AFLP fingerprinting patterns, there were 1.5 - 2 times more bands in AFLP-SSCP. If the frequencies of polymorphic bands between AFLP and AFLP-SSCP were different, it means that the polymorphic bands of AFLP-SSCP were generated by SNPs differences inside the bands (Figure 1). Dried gels were lined up using nicks to isolate targeted AFLP-SSCP bands. A sharp, clean razor blade was used to excise the selected piece of gel. The gel was washed by $\mathrm{ddH} 2 \mathrm{O}$ and then transfer to a $0.2 \mathrm{ml}$ tube with $50 \mathrm{ul}$ $\mathrm{ddH}_{2} \mathrm{O}$. The band was eluted from the gel by incubation at $4{ }^{\circ} \mathrm{C}$ for 24 hours. The tubes were centrifuged at $14,000 \mathrm{rpm}$ for $5 \mathrm{~min}$, and then the supernatant was transferred to a new tube for PCR. The PCR amplifications were conducted on $1 \mathrm{ul}$ of supernatant in $20-\mathrm{mL}$ reactions with the following composition: $1 \times$ Go Taq Flexi buffer, $2.0 \mathrm{mM} \mathrm{MgCl}_{2}, 0.2 \mathrm{mM}$ each dNTP, 2 pmol each of corresponding selective AFLP primers and 0.1 units Taq (Promega). Cycling parameters were $94^{\circ} \mathrm{C}$ for 3 min, followed by 35 cycles of $94^{\circ} \mathrm{C}$ for $1 \mathrm{~min}, 52^{\circ} \mathrm{C}$ for 1 min, and $72^{\circ} \mathrm{C}$ for $1 \mathrm{~min}$, followed by $72^{\circ} \mathrm{C}$ for $2 \mathrm{~min}$. The final products were checked by using $2 \%$ agarose gel electrophoresis and purified with QIAquick PCR purification kit (Qiagen, USA). Single strand sequencing was done either with EcoRI-core primer or MseI-core primer by sequencer ABI 3100 (Applied BioSystems, USA). Finally, the sequence data were alignment and checked by BioEdit 7.0 [14].

\section{Results}

The seven pairs of primer sets yielded a total of 142 scorable bands (size range 100 - $400 \mathrm{bp)}$ ). Eighty-five bands (59.9\%) were monomorphic, and the other 57 polymorphic. Three AFLP selective amplified products (E-ATG/ M-CTA; E-ATC-M-CGT and E-ACT/M-CGC) which contained more monomorphic bands (88.9\%, 91.7\% and 88.9\%) were used in AFLP-SSCP. These three AFLP selective amplified products yielded a total of 35 monomorphic bands in 39 scorable bands. Fifteen informative AFLP-SSCP bands were obtained from 35 monomorphic bands (excluding the bands were unclear, too closed to other bands and the frequency below than 5\%). Fourteen of 15 AFLP-SSCP bands were success re-amplification and only one band failure to get clear and specific band (Table 1; Figure 2). Ten of 14 re-amplification bands were success to get sequences (Table 1).

\section{Discussion}

In this study, we demonstrate the AFLP-SSCP method is useful to find the SNPs, and increased the detection ability of polymorphism bands (Figure 1, Figure 3). Although low genetic variation among different stocks of the Asian Seabass was detected, fifteen informative AFLP-SSCP bands were obtained from 35 monomorphic bands. Standard AFLP procedure could reflect genetic variation through: 1) SNPs within restriction sites (EcoRI: 6 bp and MseI: 4 bp); 2) SNPs within selective primer sites (EcoRI-ANNN: 1 - 4 bp and MseI-CNNN: 1 - 4 bp) and 3) SSR, deletion and insertion within AFLP bands (Figure 3). Genetic variation was detected within 16 bp (restriction sites + selective primer sites) among 39 times (loci; AFLP bands) in standard AFLP procedure. However, AFLP bands (band size: 100 - 400) containing SNPs could be further identified by AFLP-SSCP. It means genetic variation could be detected within 100 - 400 bp (AFLP bands) among 35 times (monomorphic loci). 
(a) Denaturing polyacrylamide gels (standard AFLP)

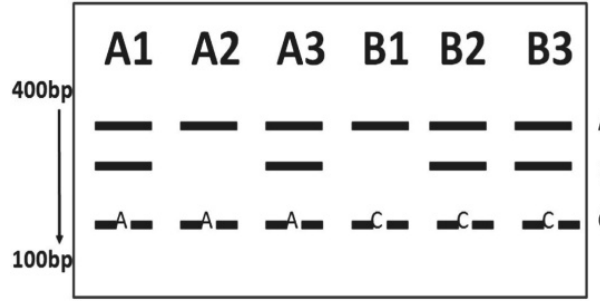

(b) Non-denaturing polyacrylamide gels (AFLP-SSCP)

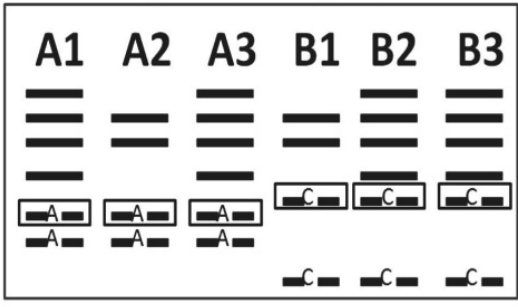

(c)

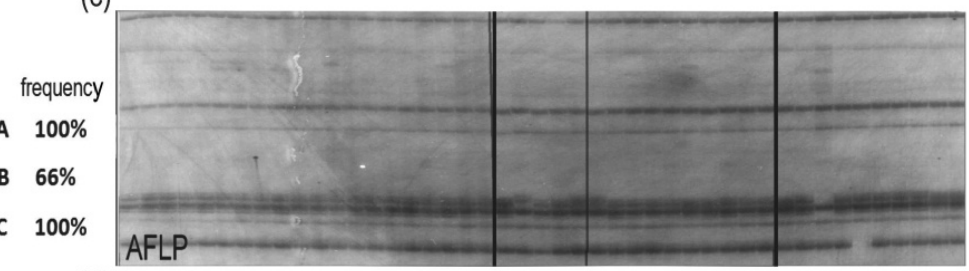

(d)

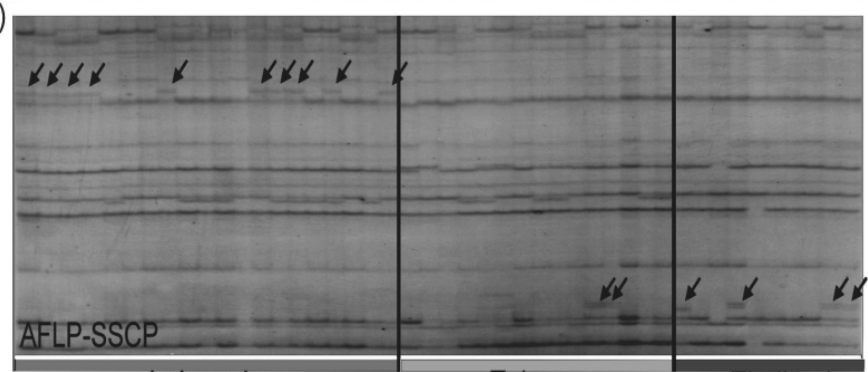

Figure 1. The profile of AFLP and AFLP-SSCP fingerprinting patterns. The PCR products were electrophoresed on 5\% denaturing polyacrylamide gels in (a) and (c) (standard AFLP) and were electrophoresed on 6\% non-denaturing polyacrylamide gels in (b) and (d) (AFLP-SSCP), respectively. The band C including SNP was monomorphic in (a), but polymorphic in (b). By comparison of bands frequency, the bands including SNPs could be found. Forty-five samples of Asian Seabass from three different stocks (Indonesia, Taiwan and Thailand) were used in AFLP (c) and AFLP-SSCP (d) analysis. No useful marker for stock discriminating in AFLP fingerprint profile (c); in contrast, two markers (black arrow) were found in AFLP-SSCP fingerprint profile (d). The polymorphic AFLP-SSCP bands were generated by SNPs differences.
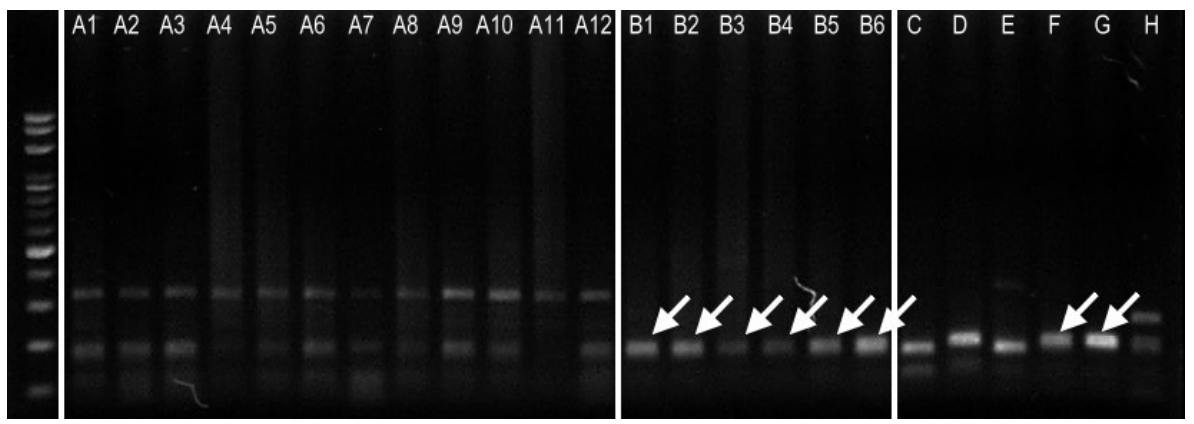

Figure 2. The profile of re-amplification bands from AFLP-SSCP bands. Lanes A1 - A12 and Lanes B1 - B6 were obtained from the same AFLP-SSCP band but different individuals, respectively. Lanes C - H were garnered from different AFLP-SSCP bands. The white arrows indicate the specific amplifications.

\begin{tabular}{ll} 
Table 1. Summary of SNPs discovered in sequenced from AFLP-SSCP method. & \\
\hline No. of total AFLP bands & 39 \\
No. of monomorphic AFLP bands & 35 \\
No. of informative AFLP-SSCP bands & 15 \\
No. of success of re-amplification from AFLP-SSCP bands & 14 \\
Success of sequencing bands & 10 \\
\hline
\end{tabular}

Therefore, useless AFLP markers (low polymorphic and monomorphic bands) could increase detection ability of genetic variation through AFLP-SSCP analysis (Figure 3).

Fifteen informative AFLP-SSCP bands were generated from 35 monomorphic AFLP bands. Although the percentage of informative AFLP-SSCP bands (42.9\%) was not high, it guaranteed the success of "informative" SNPs discovering from the sequences. When SNPs are rare (below 5\%) in the analysis samples, they are not 


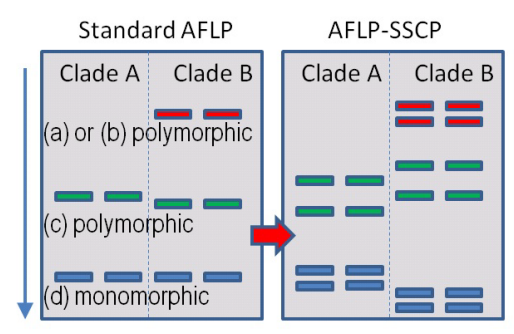

(a) SNPs within restriction sites (10 bp)

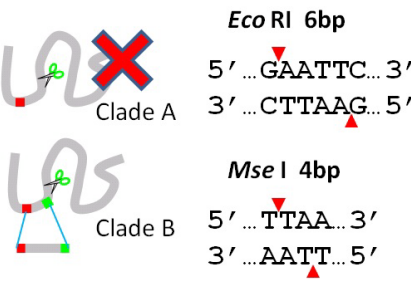

(b) SNPs within selective primer sites (2-8 bp) (c) SSR, deletion, insertion
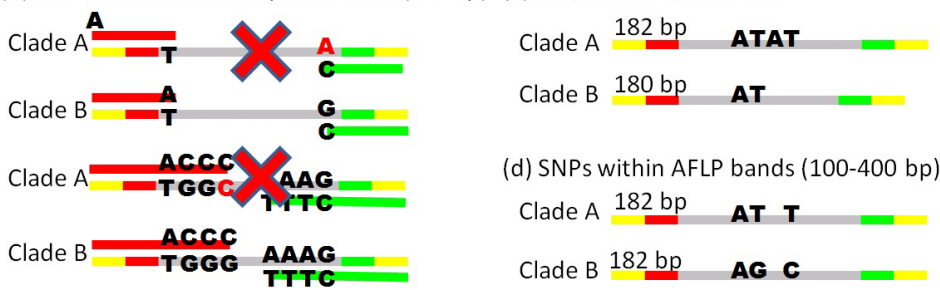

(d) SNPs within AFLP bands (100-400 bp) Clade A 182 bp AT T Clade B 182 bp AG C

Figure 3. Four genetic variation types of standard AFLP and AFLP-SSCP. Genetic variation in AFLP and AFLP-SSCP: (a) SNPs within restriction sites (EcoRI: 6 bp and MseI: 4 bp); (b) SNPs within selective primer sites (EcoRI-ANNN: 1 - 4 bp and MseI-CNNN: 1 - 4 bp); (c) SSR, deletion and insertion within AFLP bands; (d) SNPs within AFLP bands (band size: 100 - 400). (a), (b) and (c) were polymorphic in standard AFLP and AFLP-SSCP. (d) was monomorphic in standard AFLP but polymorphic in AFLP-SSCP.

considered as "informative”. Additionally, all mutations could not be detected by using one electrophoresis condition in SSCP analysis [15]. There are many parameters that have been found empirically to affect the sensitivity of SSCP analysis [15]. Therefore, more mutations can be detected by a combination of 2 - 3 different conditions [15].

Fourteen of 15 AFLP-SSCP bands were success re-amplification and only one band failure to get clear and specific band (Table 1; Figure 2). The non-specific amplification easily presented in this step, but it usually would be saved by cutting and re-amplifying from multiple copies (each AFLP-SSCP band was cut and re-amplified from at least 5 individual bands). Ten of 14 re-amplification bands were success to get sequences (Table 1). One of the non-specific amplification and 4 failure sequencing re-amplification bands might cause by the multiple size homoplasy templates. Caballero et al. (2008) [16] report that average $10 \%$ to $15 \%$ of homoplasy existed in the AFLP analysis, and the significant impact of AFLP homoplasy on its effectiveness. In our experience, using one or two plus four nucleotides selective primers (EcoRI-ANNN and MseI-CNNN) were useful to reduce the AFLP homoplasy.

In the case of directly sequencing of the AFLP-SSCP bands, $71.43 \%$ (10 of 14) were generated. Good quality and identical sequence data were obtained for at least 2 additional individual. The size range of the sequenced bands was between 100 - $400 \mathrm{bp}$. The ten sequences were long enough to design primers, and could be used to re-amplify and sequenced for the genomic DNA sample. These results show that the directly sequencing is available for the conversion of AFLP-SSCP markers into simple PCR markers without cloning. It is also reported by Nicod and Largiader (2003) [4].

\section{Conclusion}

In this study, we present a cheap and convenient AFLP-based SNPs discovery strategy that is highly efficient for developing informative SNP markers without any prior information. We solved the problem of directly sequencing from AFLP markers that sometimes are converted to monomorphic fragments. These may be due to loss of the uniqueness of the primer-binding site on which the polymorphism was based. Using the AFLP-SSCP method, more polymorphisms were detected. It was especially useful in the high homozygous species or strains. Although more and more high-throughput techniques were developed, the AFLP still show the advanced in nonmodel organisms. The AFLP-based techniques provide the cheap and convenient tools for the short-term research (standard AFLP) and SNPs discovering (AFLP-SSCP). This novel strategy not only enhances the application range of AFLP but also makes the connection between AFLP with SNPs. 


\section{Acknowledgements}

We thank Dr. C.-L. Chang (Fisheries Research Institue, TAIWAN) for providing samples and Dr. Z.-Y. Wang (Ji-Mei University, Xiamen, PROC) for technical advice. The work described in this paper was fully supported by grants from the center for Marine Bioscience and Biotechnology (CMBB), National Taiwan Ocean University, TAIWAN.

\section{References}

[1] Morin, P.A., Luikart, G., Wayne, R.K. and Grp, S.W. (2004) SNPs in Ecology, Evolution and Conservation. Trends in Ecology \& Evolution, 19, 208-216. http://dx.doi.org/10.1016/j.tree.2004.01.009

[2] Kim, S. and Misra, A. (2007) SNP Genotyping: Technologies and Biomedical Applications. Annual Review of Biomedical Engineering, 9, 289-320. http://dx.doi.org/10.1146/annurev.bioeng.9.060906.152037

[3] Garvin, M.R., Saitoh, K. and Gharrett, A.J. (2010) Application of Single Nucleotide Polymorphisms to Non-Model Species: A Technical Review. Molecular Ecology Resources, 10, 915-934. http://dx.doi.org/10.1111/j.1755-0998.2010.02891.x

[4] Nicod, J.C. and Largiadèr, C.R. (2003) SNPs by AFLP (SBA): A Rapid SNP Isolation Strategy for Non-Model Organisms. Nucleic Acids Research, 31, e19. http://dx.doi.org/10.1093/nar/gng019

[5] Bensch, S. and Akesson, M. (2005) Ten Years of AFLP in Ecology and Evolution: Why So Few Animals? Molecular Ecology, 14, 2899-2914. http://dx.doi.org/10.1111/j.1365-294X.2005.02655.X

[6] Meudt, H.M. and Clarke, A.C. (2007) Almost Forgotten or Latest Practice? AFLP Applications, Analyses and Advances. Trends in Plant Science, 12, 106-117. http://dx.doi.org/10.1016/j.tplants.2007.02.001

[7] Liu, Z.J. and Cordes, J.F. (2004) DNA Marker Technologies and Their Applications in Aquaculture Genetics. Aquaculture, 238, 1-37. http://dx.doi.org/10.1016/j.aquaculture.2004.05.027

[8] Shan, X., Blake, T.K. and Talbert, L.E. (1999) Conversion of AFLP Markers to Sequence-Specific PCR Markers in Barley and Wheat. Theoretical and Applied Genetics, 98, 1072-1078. http://dx.doi.org/10.1007/s001220051169

[9] Meksem, K., Ruben, E., Hyten, D., Triwitayakorn, K. and Lightfoot, D.A. (2001) Conversion of AFLP Bands into High-Throughput DNA Markers. Molecular Genetics and Genomics, 265, 207-214. http://dx.doi.org/10.1007/s004380000418

[10] Brugmans, B., van der Hulst, R.G.M., Visser, R.G.F., Lindhout, P. and van Eck, H.J. (2003) A New and Versatile Method for the Successful Conversion of AFLP Markers into Simple Single Locus Markers. Nucleic Acids Research, 31, e55. http://dx.doi.org/10.1093/nar/gng055

[11] Zhang, Z.Z., Guo, M.L. and Zhang, J.D. (2009) Identification of AFLP Fragments Linked to Hydroxysafflor Yellow A in Flos Carthami and Conversion to a SCAR Marker for Rapid Selection. Molecular Breeding, 23, 229-237. http://dx.doi.org/10.1007/s11032-008-9228-9

[12] Vos, P., Hogers, R., Bleeker, M., et al. (1995) AFLP-A New Technique for DNA Fingerprinting. Nucleic Acids Research, 23, 4407-4414. http://dx.doi.org/10.1093/nar/23.21.4407

[13] Wang, Z.Y., Tsoi, K.H. and Chu, K.H. (2004) Applications of AFLP Technology in Genetic and Phylogenetic Analysis of Penaeid Shrimp. Biochemical Systematics and Ecology, 32, 399-407. http://dx.doi.org/10.1016/j.bse.2003.10.006

[14] Hall, T.A. (1999) BioEdit: A User-Friendly Biological Sequence Alignment Editor and Analysis Program for Windows 95/98/NT. Nucleic Acids Symposium Series, 41, 95-98.

[15] Nataraj, A.J., Olivos-Glander, I., Kusukawa, N. and Highsmith, W.E. (1999) Single-Strand Conformation Polymorphism and Heteroduplex Analysis for Gel-Based Mutation Detection. Electrophoresis, 20, 1177-1185. http://dx.doi.org/10.1002/(SICI)1522-2683(19990101)20:6<1177::AID-ELPS1177>3.0.CO;2-2

[16] Caballero, A., Quesada, H. and Rolán-Alvarez, E. (2008) Impact of Amplified Fragment Length Polymorphism Size Homoplasy on the Estimation of Population Genetic Diversity and the Detection of Selective Loci. Genetics, 179, 539554. http://dx.doi.org/10.1534/genetics.107.083246 
Scientific Research Publishing (SCIRP) is one of the largest Open Access journal publishers. It is currently publishing more than 200 open access, online, peer-reviewed journals covering a wide range of academic disciplines. SCIRP serves the worldwide academic communities and contributes to the progress and application of science with its publication.

Other selected journals from SCIRP are listed as below. Submit your manuscript to us via either submit@scirp.org or Online Submission Portal.
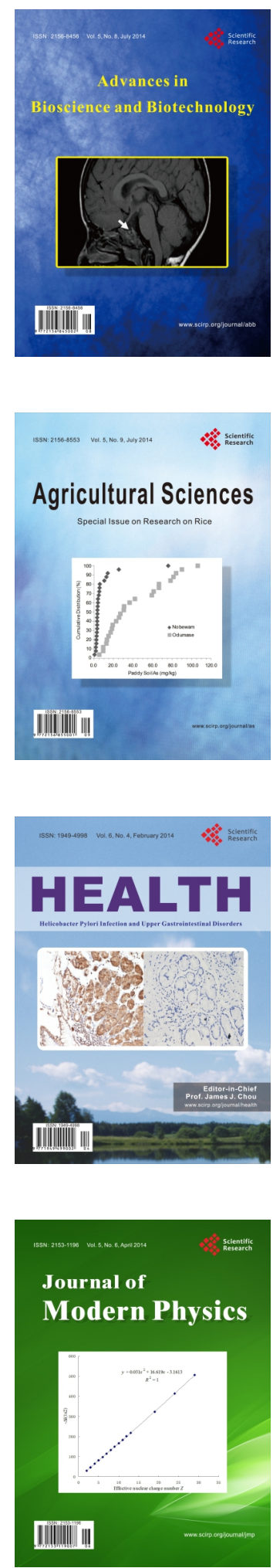
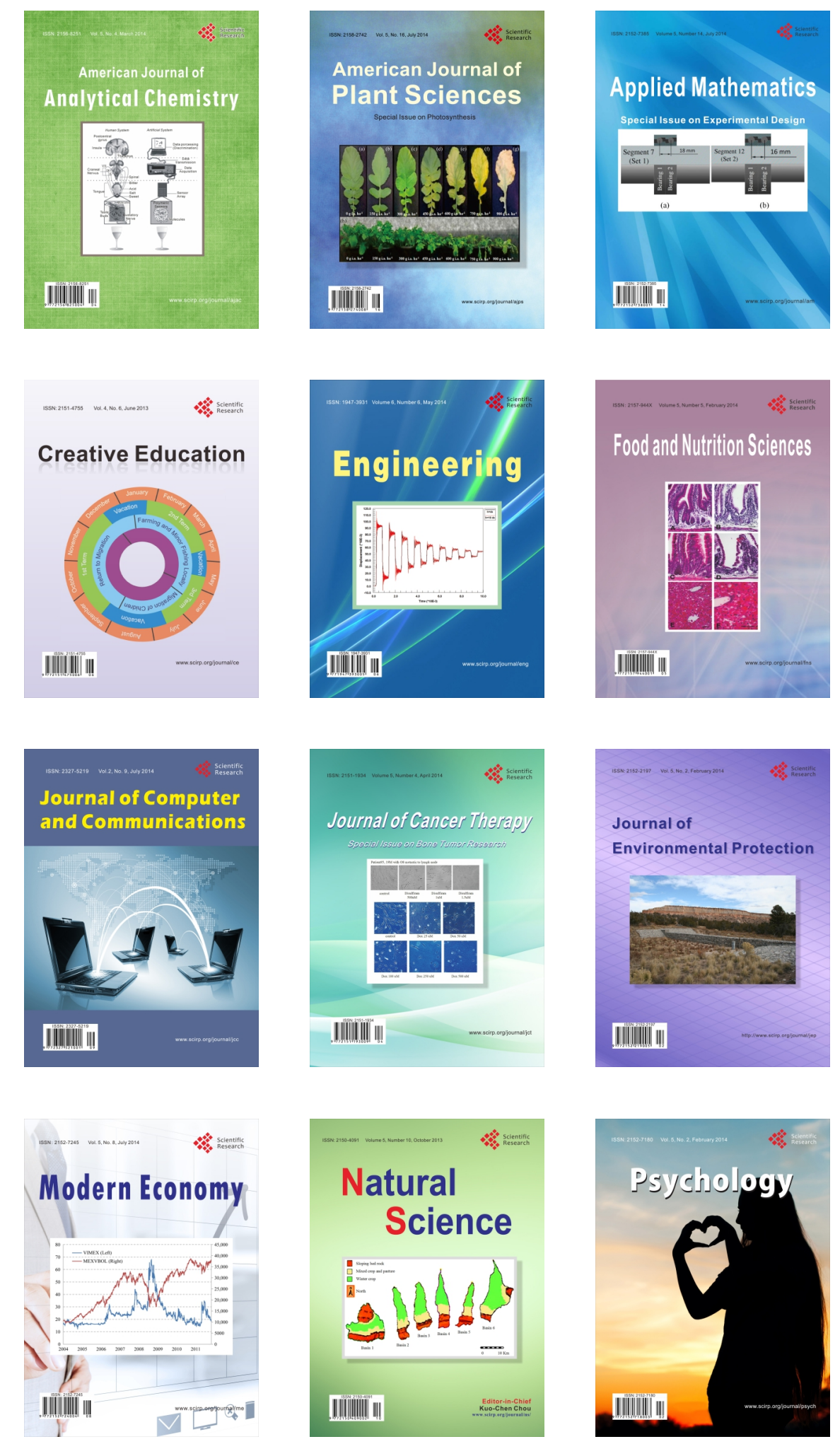